\title{
Noninvasive Diagnosis of Allograft Vascular Disease after Heart Transplantation
}

\author{
Fernando Bacal, N oedir Antonio Groppo Stolf, Viviane Cordeiro Veiga, W illiam A. Chalela, \\ Cesar Grupi, Ana Clara Rodrigues, Eulógio E. Martinez, A lfredo Inácio Fiorelli, Luiz Felipe Pinho Moreira, \\ Edimar Alcides Bocchi, Giovanni Bellotti, José Antonio Franchini Ramires
}

São Paulo, SP - Brazil

Objective - To determine the predictive values of noninvasive tests for the detection of allograft vascular disease.

Methods - We studied 39 patients with mean ages of $48 \pm 13$ years and a follow-up period of $86 \pm 13$ months. The diagnosis of allograft vascular disease was made by cinecoronary arteriography, and it was considered as positive if lesions existed that caused $\geq 50 \%$ obstruction of the lumen. Patients underwent $24 \mathrm{~h}$ Holter monitoring, thallium scintigraphy, a treadmill stress test, and dobutamine stress echocardiography. Sensitivity, specificity, and positive and negative predictive values were determined in percentages for each method, as compared with the cine-coronary arteriography results.

Results - Allograft vascular disease was found in 15 (38\%) patients. The Holter test showed $15.4 \%$ sensitivity, 95.5\% specificity. For the treadmill stress test, sensitivity was $10 \%$, specificity was $100 \%$. When thallium scintigraphy was used, sensitivity was $40 \%$, specificity $95.8 \%$. On echocardiography with dobutamine, we found a $63.6 \%$ sensitivity, $91.3 \%$ specificity. When the dobutamine echocardiogram was associated with scintigraphy, sensitivity was $71.4 \%$, specificity was $87 \%$.

Conclusion - In this group of patients, the combination of two noninvasive methods (dobutamine echocardiography and thallium scintigraphy) may be a good alternative for the detection of allograft vascular disease in asymptomatic patients with normal ventricular function.

Key words: heart transplantation, allograft vascular disease, diagnostic methods

Instituto do Coração do Hospital das Clínicas - FMUSP

Mailing address: Fernando Bacal - InCor - Av. Dr. Enéas C. Aguiar $44-$

05403-000 - São Paulo, SP - Brazil
Allograft vascular disease is the main late complication in the follow-up of patients who undergo heart transplantation.

Ever since the experimental period of heart transplantation, Lower et al ${ }^{1}$ described coronary atherosclerosis in a dog that underwent heart transplantation and proved that this complication could affect the late post-transplantation follow-up, yet the first description of coronary disease affecting human grafts was made by Thomson et $\mathrm{al}^{2}$.

Allograft vascular disease is at this time responsible for most of the deaths that occur during the late posttransplantation follow-up, and its incidence amounts to an estimated $10 \%$ per postoperative year, reaching $40 \%$ to $50 \%$ in receptors by completion of their $5^{\text {th }}$ postoperative year ${ }^{3-7}$.

The first manifestation of the disease is often congestive heart failure or even sudden death, arising from an asymptomatic acute infarction of the myocardium, since hearts are denervated and have therefore no afferent sensitive fibers. In cases of coronary failure, the typical precordial pain may not be reported, and only a few reports with a documented partial reinnervation have been published ${ }^{8-10}$.

Another important aspect concerns the diagnosis of vascular disease of the graft. The method still commonly used is an annual angio-coronary angiography after the first postoperative year. Gao et al ${ }^{11}$ proposed a classification based on the characteristics, location, and extension of the coronary lesions, defining them by their characteristics as diffuse, multiarterial, and predominantly distally affecting lesions. On angiography, coronary lesions are classically defined as type A, B, and C: type A - discrete proximal, medial, or distal lesions in major arteries or their branches; type $\mathrm{B}$ - diffuse, concentric lesions involving the medial and distal thirds of major arteries or their branches; type $\mathrm{C}$ - association between types A and B.

The complementation of this test with the information obtained from intravascular ultrasound has allowed a more accurate definition of coronary lesions, mainly in cases that 
were underestimated by arteriographic analysis. Intravascular ultrasound can also document endothelial alterations occurring early after transplantation, when the media and intima of the blood vessels and the endothelial response to the use of certain drugs with vasoactive properties are analyzed separately ${ }^{12-14}$. Despite the potential benefit of this method for the detection of vascular disease of the graft, with high sensitivity and specificity, its applicability in Brazil is still limited, due to the small number of medical units that have it and its high cost. Noninvasive methods for the diagnosis of vascular disease of the graft have been studied in an attempt to replace the annual angiographic analysis, but the results are still controversial. Some groups have reported good diagnostic sensitivity with tests such as myocardial scintigraphy and stress echocardiography, but the great majority go on using annual arteriography due to the low sensitivity and specificity of those methods in their series of patients ${ }^{15-18}$.

The objective of this study was to compare the noninvasive diagnosis of vascular disease in asymptomatic patients with normal ventricular function, thus characterizing a population with a low suspicion of this disease.

\section{Methods}

We studied 39 patients who underwent orthotopic heart transplantation at the Instituto do Coração do Hospital das Clínicas da Faculdade de Medicina da USP, with a mean age of $48 \pm 13$ years, and a mean follow-up period of $86 \pm 31$ months.

The inclusion criteria were: over two years of followup, normal ventricular function on echocardiogram at rest, absence of symptoms of angina or heart failure, absence of acute rejection, and compliance with the study protocol.

The following tests were performed: cine-coronary arteriography, 24-hour Holter monitor, treadmill stress test, myocardial thallium scintigraphy under physical stress (ergometric test) or pharmacological stress (dipyridamole) in patients with exercise limitations or patients with permanent pacemakers, stress echocardiography with dobutamine. All the noninvasive tests were performed within the same week of the study.

The technique adopted for coronary angiography was that described by Sones and Shirey ${ }^{19}$. Philips equipment model Optimus 1050 with a 6.5-to-9-inch image intensifier coupled with an Arritecno camera with a shooting rate of 30 pictures per second was used. The films were separately analyzed by two specialists of the InCor Hemodynamics service, who had no previous knowledge of the patients' clinical and laboratory data. Each artery was analyzed, in an attempt to establish the degree of lumen reduction of the vessel on at least two incidences: oblique anterior right and oblique anterior left. This method was considered the gold standard for the definition of the presence or absence of vascular disease of the graft. The test was considered positive for vascular disease of the graft whenever the lumen obstruction was $\geq 50 \%{ }^{20,21}$, after which the designations disease "Yes" or "No"criteria were adopted. Lesions <50\% were considered wall irregularities and disregarded. Based on the results of this method, sensitivity, specificity, and the positive and negative predictive values of the noninvasive diagnostic methods were calculated.

With regard to 24-hour electrocardiography (Holter), patients were monitored by means of a Marquette 8000 twochannel portable monitor with modulated amplitude wave recording from Marquette (Milwaukee, WI, USA), containing a cassette tape sufficient for a continuous 24-hour study. The monitor was connected to the patient the moment he or she entered the study, and patients were instructed to report the occurrence of any possible symptoms during these 24 hours, in order to have them recorded in an attached diary.

Once the monitor was removed, the tapes were analyzed by a Marquette Laser SXP processor (1988) from Marquette (Milwaukee, WI, USA) and evaluated by the staff of the Holter service. Heart beats were classified and evaluated manually. For the diagnosis of ischemic events, an isoelectric point - the PQ interval - and a point $60 \mathrm{~m}$ distant from the $\mathrm{J}$ point were manually adjusted upon the automatic reading, in determine the baseline of the ST segment, starting from which the variations upwards, up-unlevelling, and downwards, down-unlevelling, were considered.

To be diagnosed as an ischemic event, an elevation $\geq 2 \mathrm{~mm}$ or a horizontal or descending depression $\geq 1 \mathrm{~mm}$ of the ST segment from the baseline had to occur, lasting at least one minute, and returning to the baseline for at least one minute. The possible ischemic events identified in this manner were defined as ischemic episodes (positive test), and each one of them was recorded on paper, at a $25 \mathrm{~mm} / \mathrm{s}$ speed, with a $1 \mathrm{mV}$ standard, using a Marquette laser printer, from which the variations of the ST segment were manually reevaluated.

The exercise stress tests were performed according to criteria established by the Consenso Nacional de Ergometria (National Stress Test Consensus) ${ }^{22}$.

Exercise was interrupted when the patient attained the heart rate considered the maximum, obtained by the formula: maximum $H R=210$-(agex 0.65$)$, corresponding to $100 \%$ of the patient's heart rate during stress. Whenever this parameter was not reached, the test interruption criteria were the ones established by the American Heart Association ${ }^{23}$.

A MAT 2100 treadmill with a movable ramp was used, coupled with an ML 8000 Stress System from Fukuda Denshi Co. Ltd. The protocol used was Ellestad's with a recording of 12 simultaneous classical derivations.

Blood pressure measurements were automatically made every $60 \mathrm{~s}$ by a Colin device, model STBP 780, coupled with the ML 8000 software.

A test was considered to be positive or ischemic if a down-unlevelling occurred of the ST segment with a horizontal or descending morphology, or both, larger or equal to $1.0 \mathrm{~mm}$ for men and $2.0 \mathrm{~mm}$ for women; a down-unlevelling of the ascending ST segment with the point $\mathrm{Y} \geq 2.0 \mathrm{~mm}$ for men and 3.0mm for women. 
The radioactive drug used in myocardial perfusion scintigraphy was thallium-201 chloride $\left({ }^{201} \mathrm{TI}\right)$. The perfusion study protocol encompassed two phases: 1) images were taken immediately after radionuclide injection on the peak of exercise. The injected dose was $111 \mathrm{MBq}$, and tomography images were obtained with the patient in dorsal decubitus; 2) Four hours later and in the same way, redistribution images were obtained.

To obtain the tomography images, a Siemens scintillation camera, model Orbiter ZLC-Digitrac 750 was used, coupled with a Maxdelta computer (Microvax-3300). After the reconstruction of the images, transversal sections were obtained at every $6.09 \mathrm{~mm}$, reoriented to the heart axis, thus allowing the determination of the sections corresponding to the three planes mutually perpendicular to the system of coordinates set for the heart.

For the interpretation of the images, the heart was divided into five regions: septal, anterior, inferior, apical, and lateral. A value was attributed to each one of them by means of a random grade system, according to the uptake of the radioactive drug $(0=$ normal uptake; $1=$ discrete underuptake; $3=$ pronounced underuptake). Uptake (perfusion) defects were considered if observed on at least two different axes and two sequential sections. A decrease of at least one point of this grading in the analyzed region, when the images of physical stress are compared with the other phases, was interpreted as a transient underuptake. It was considered as partial if the value zero was not observed on the redistribution the reinjection, the rest images, or all of these. The maintenance or increase of this gradation was called persistent underuptake (fibrosis). Once transient underuptake was documented, the test was defined as positive for the detection of vascular disease of the graft.

The protocol for dobutamine stress echocardiography consisted of continuous dobutamine administration through an infusion pump, starting with a $10 \mathrm{mcg} / \mathrm{kg} / \mathrm{min}$ dose for 3 minutes, progressively increasing to 20,30 , and $40 \mathrm{mcg} / \mathrm{kg} / \mathrm{min}$, until the patient attained the submaximum heart rate for his or her age ( $85 \%$ of the maximum heart rate). If this did not occur, atropin was given in $0.25 \mathrm{mg}$ boli up to a total dose of $1 \mathrm{mg}$. The reason for anticipating the end of the test was the occurrence of side effects, such as an increase in systolic blood pressure to more than $220 \mathrm{mmHg}$, complex arrhythmia or important symptoms of intolerance to medication (headache, angina). Neither hypotension (a drop of systolic blood pressure $>20 \mathrm{mmHg}$ with respect to the base level) nor the occurrence of segmental contractility alterations were considered as reasons to discontinue the protocol. Bidimensional echocardiograms were performed by means of an ATL echocardiograph model 3000 or 5000 and a 3.5 or $2.5 \mathrm{MHz}$ transductor. Images were obtained at rest, at the end of stage 10 and 40 of dobutamine administration, and upon recovery. The whole test was recorded on videocassette, and the data were stored on a computer for processing and ulterior analysis. Four basic images were obtained: parasternal long axis and short axis, apical two cameras and four cameras, and they were analyzed by two observers with no knowledge about the cine-coronary arteriography and, whenever there was a disagreement, an evaluation by a third observer was requested. The result was considered as positive if any alteration of in regional wall motion appeared. The wall motion index was given by the following values: 1 ) normal: an increase in wall motion and systolic thickening after dobutamine; 2) hypokinesia: a decrease of systolic thickening and wall motion during dobutamine; 3) akinesia: the absence of thickening during dobutamine, and 4) dyskinesia: an outward movement of the analyzed segment after dobutamine. The 12-derivation electrocardiogram was made at rest and after the maximum dobutamine dose, and blood pressure was constantly monitored, being recorded at the end of each dobutamine stage $^{24}$.

Statistical analysis - In order to comparatively assess the noninvasive tests with the gold standard, catheterization, for the diagnosis of coronary disease, sensitivity and specificity indexes, as well as the positive and negative predictive values, were calculated. To evaluate whether the disagreement proportion between each test and catheterization was negligible, i. e., close enough to zero, a test for generalized linear models, based on Wald's statistics ${ }^{25}$, was used.

The level of significance established for the analysis was 5\%, and all calculations were made according to the SAS (Statistical Analysis System) ${ }^{26}$.

\section{Results}

In $15(38.4 \%)$ of the 39 studied patients, cine-coronary arteriography showed a vascular disease of the graft, characterized by a $\geq 50 \%$ obstructive coronary lesion, in at least one vessel. Fifteen patients were studied during the period from the $2^{\text {nd }}$ to the $5^{\text {th }}$ postoperative year, 17 from the $6^{\text {th }}$ to the $9^{\text {th }}$ year and seven after more than 10 years from the operation. As for the characteristics of the coronary lesions found, five were triarterial, seven were biarterial and three were uniarterial. From the viewpoint of Gao et al's ${ }^{11}$ arteriographic classification, 11 patients presented with type $\mathrm{C}$ lesions, two patients with type $\mathrm{A}$, and two patients with type B.

Thirty-five patients had the 24-hour electrocardiogram (Holter) carried out. Excluded from the analysis were those who had a block of the D branch and those who wore a pacemaker, due to the difficulties in analyzing the alterations of ventricular repolarization. Ischemia was the result found in three patients, two of whom had vascular disease of the graft. Of the 32 patients with normal results, 21 did not have the disease and 11 had it. The sensitivity of the test was $15.4 \%$, specificity was $95.5 \%$, positive predictive value was $66.7 \%$, and negative predictive value was $65.6 \%$ (Table I).

Thirty-one patients underwent electrocardiography under stress (TE). Patients with a permanent pacemaker and those who had physical limitations disabling them from exercise were excluded. Only one patient had a positive result by this method, and he had a vascular disease of the graft. Of 


\begin{tabular}{|c|c|c|c|c|c|c|}
\hline \multirow[b]{2}{*}{ Test } & \multirow[b]{2}{*}{ Result } & \multicolumn{2}{|c|}{ Disease } & \multirow[b]{2}{*}{ Total } & \multirow{2}{*}{$\begin{array}{c}\text { Sensitivity } \\
\text { Especificidade }\end{array}$} & \multirow{2}{*}{$\begin{array}{l}15.4 \% \\
95.5 \%\end{array}$} \\
\hline & & No & Yes & & & \\
\hline \multirow[t]{3}{*}{ Holter } & No & 21 & 11 & 32 & $\%$ Concordance & $65.7 \%$ \\
\hline & Yes & 1 & 2 & 3 & $\%$ Discordance & $34.3 \%$ \\
\hline & Total & 22 & 13 & 35 & $\begin{array}{l}\text { Predictive value }+ \\
\text { Predictive value - }\end{array}$ & $\begin{array}{l}66.7 \% \\
65.6 \%\end{array}$ \\
\hline
\end{tabular}

the 30 patients with a normal result, 21 did not have the disease and nine had it. The sensitivity of this test was 10\%, its specificity was $100 \%$, its positive predictive value was $100 \%$, and its negative predictive value was $70 \%$ (Table II).

All patients underwent scintigraphy with thallium-201 (Thallium). Seven patients had a positive test result, and six of them had a vascular disease of the graft. Of the 32 patients with normal results, nine had a vascular disease of the graft, as documented by cine-coronary arteriography. The sensitivity of the test was $40 \%$, its specificity was $95.8 \%$, its positive predictive value was $85.7 \%$, and its negative predictive value was $71.9 \%$ (Table III).

Thirty-four patients underwent stress electrocardiography with dobutamine (Echo-Dobu). Those who were unable to have the test done due to technical difficulties were excluded, mainly because of the lack of an electrocardiographic window favorable for analysis. Nine patients had a positive test result, and seven of them had the disease. Of the 25 patients who tested normal, only four had a vascular disease of the graft. The sensitivity of this test was $63.6 \%$, its specificity was $91.3 \%$, its positive predictive value was $77.8 \%$, and its negative predictive value was $84 \%$ (Table IV).

A joint analysis was performed of the two diagnostic methods with the best sensitivity for the detection of vascular disease of the graft (dobutamine echocardiogra-

\begin{tabular}{|lccccccc|}
\hline \multicolumn{8}{|c|}{ Table II - Results of treadmill stress test in the detection of vascular } \\
disease of the graft
\end{tabular}

\begin{tabular}{|ccccccc|}
\hline \multicolumn{7}{|c|}{ Table III - Results of thallium scintigraphy in the detection of } \\
vascular disease of the graft
\end{tabular}

\begin{tabular}{|c|c|c|c|c|c|c|}
\hline \multicolumn{7}{|c|}{$\begin{array}{l}\text { Table IV - Results of stress echocardiography in the detection of } \\
\text { vascular disease of the graft }\end{array}$} \\
\hline \multirow[b]{2}{*}{ Test } & \multirow[b]{2}{*}{ Result } & \multicolumn{2}{|c|}{ Disease } & \multirow[b]{2}{*}{ Total } & \multirow{2}{*}{$\begin{array}{l}\text { Sensitivity } \\
\text { Specificity }\end{array}$} & \multirow{2}{*}{$\begin{array}{l}63,6 \% \\
91,3 \%\end{array}$} \\
\hline & & No & Yes & & & \\
\hline \multirow[t]{4}{*}{ Stress Echo } & No & 21 & 4 & 25 & $\%$ Concordance & $82,4 \%$ \\
\hline & Yes & 2 & 7 & 9 & \% Discordance & $17,6 \%$ \\
\hline & Total & 23 & 11 & 34 & Predictive value+ & $77,8 \%$ \\
\hline & & & & & Predictive value - & $84,0 \%$ \\
\hline
\end{tabular}

phy and thallium scintigraphy). The result was considered as positive whenever at least one of them showed ischemia. The results found are shown in Table $\mathrm{V}$ and figure 1 .

\section{Discussion}

Coronary angiography is still the gold standard for the diagnosis of presence or absence of coronary artery disease in the general population. In patients who undergo heart transplantation, the role of this test in the diagnosis of vascular disease of the graft also seems well established, and therefore an annual angiography is recommended after the first postoperative year.

The incidence of allograft vascular disease is estimated at $10 \%$ per year, amounting to a total of $40 \%$ to $50 \%$ by the end of the $5^{\text {th }}$ year after operation ${ }^{27,28}$. Cases, however, do exist where evolution is rapid, with diffuse and severe lesions even before the first year after transplantation has elapsed, and the causes of this occurrence are still obscure, although histological aspects similar to arteritis have been shown in such cases. In our analysis, we found a 38\% incidence of vascular disease of the graft documented by angiography in asymptomatic patients with normal ventricular function, thus characterizing a population with a low suspicion rate for this disease. The distribution pattern of the

\begin{tabular}{|c|c|c|c|c|c|c|}
\hline \multicolumn{7}{|c|}{$\begin{array}{c}\text { Table } \mathrm{V} \text { - Results of the association of stress echocardiography with } \\
\text { thallium scintigraphy in the detection of vascular disease of the } \\
\text { graft }\end{array}$} \\
\hline \multirow[b]{2}{*}{ Test } & \multirow[b]{2}{*}{ Result } & \multicolumn{2}{|c|}{ Disease } & \multirow[b]{2}{*}{ Total } & \multirow{2}{*}{$\begin{array}{l}\text { Sensitivity } \\
\text { Specificity }\end{array}$} & \multirow{2}{*}{$\begin{array}{l}71,4 \% \\
87,0 \%\end{array}$} \\
\hline & & No & Yes & & & \\
\hline Stress Echo & No & 20 & 4 & 24 & $\%$ Concordance & $81,1 \%$ \\
\hline \multirow[t]{3}{*}{ Thallium } & Yes & 3 & 10 & 13 & $\%$ Discordance & $18,9 \%$ \\
\hline & Total & 23 & 14 & 37 & Predictive value+ & $76,9 \%$ \\
\hline & & & & & Predictive value - & $83,3 \%$ \\
\hline
\end{tabular}

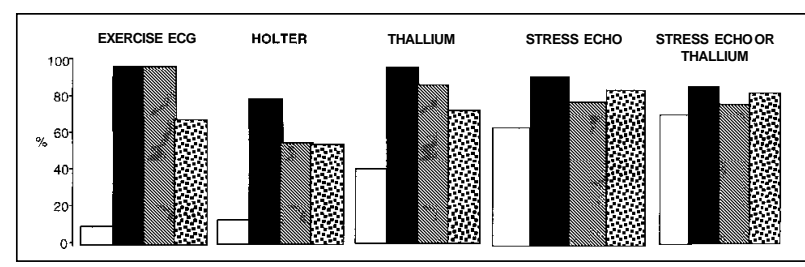

Fig. 1 - The graph shows the comparison between the detection tests for vascular disease of the graft. Sensitivity, specificity, positive predictive value, negative predictive value. 
obstructive lesions of the coronaries was similar to that found by other investigators, i. e., multiarterial, diffuse lesions, frequently affecting the distal bed. On the other hand, Uretsky et al. ${ }^{29}$ showed that angiographic analysis might underestimate the existing lesions; in their study, they documented a $28 \%$ occurrence of cardiac events in patients with a normal angiography. Some aspects have been proposed for the standardization of this method, mainly the need to use the same angle in the analysis of each study, the taking of measurements at the same point in each vessel and under the same vasomotor tonus and, preferably, the use of the computer-assisted method for a better definition of the degree of obstruction of the vessel. Analyses should be made by at least two observers and possibly by a third one, if any disagreement occurs between the first ones. Currently, with the additional information given by the intravascular ultrasound, lesions can be better defined, especially when they are discrete. Early studies with this method, made as soon as within the first weeks after transplantation, allowed the identification of endothelial dysfunctions, probably due to immunological alterations, which may predispose in the future to the occurrence of vascular disease of the graft ${ }^{29-31}$. As already mentioned, the characteristic coronary lesions are diffuse, affecting main and secondary branches, with a frequent distal involvement, making therapeutic propositions such as angioplasty and surgical myocardial revascularization very difficult. In practice, doctors are faced with a big dilemma, i. e., to know that the patient has a severe coronary affliction and to be, so far, unable to intervene effectively both in prevention and in treatment. In cases where the diffuse coronary affliction is accompanied by ventricular dysfunction, the therapeutic indication is a new transplant, which has given poorer results than the first transplant in a number of series in the literature ${ }^{6,7}$. This fact, associated with low organ availability, leads us to ponder about this indication, even from an ethical point of view, because the chance of the first transplant can be given to patients who have been on the waiting list longer. The use of noninvasive diagnostic methods will become more important if, in the future, their effectiveness in detecting patients at greater risk of developing cardiac events further on will be proven, thus allowing detection of those with severer conditions, who will need an invasive diagnostic study.

The use of stress electrocardiography for the detection of ischemia in conventional coronary patients is well established; yet, its usefulness in patients who undergo cardiac transplantation is still controversial. Reports of other authors have shown low sensitivity and specificity of this method, which corresponds to the findings of this study ${ }^{32,33}$. In this series, the sensitivity of the method was only $10 \%$, which makes it difficult to use in this specific group of patients on a daily medical practice basis. Some factors seem to have an influence on these results. First of all, given the fact that the transplanted heart is denerved, the lack of innervation has an influence on the physiological response of the subject to exercise, so mainly a low chronotropic response is observed, associated with the presence of high ventricular filling pressures and increased levels of circulating catecholamines, attempting to compensate for that chronotropic deficiency. The alterations described here, together with blood hypertension that often accompanies the patients, may lead to diastolic dysfunction, impairing the tolerance of transplanted individuals to stress. In practice, patients show difficulties with their heart rate response, and this represents a limitation of the method for the detection of ischemia, because the triggering of ischemic events upon physical stress depends directly on the increase of the heart rate.

The use of a 24-hour electrocardiogram (Holter) as a diagnostic method for ischemic events seems to be well established, especially for the detection of the so-called silent ischemia, defined as the occurrence of ischemic events with no medical expression that might identify them. In studies with patients with coronary disease, most of the ischemic events identified by Holter are said to be asymptomatic ${ }^{34,35}$. Patients who undergo cardiac transplantation fit the model of silent ischemia perfectly, because their denerved hearts have no afferent sensitive fibers, and the first manifestation of coronary failure is frequently sudden death or even a picture of heart failure. Yet, in practice, Holter use in patients who undergo heart transplantation has shown a low diagnostic sensitivity. In this series, we found a $15 \%$ sensitivity, with most patients being able to have the test done, except for those who had permanent pacemakers or a block of the right branch who had to be excluded because of the difficulties in analyzing the ventricular repolarization. Some important aspects are worth considering in the attempt to explain our results. Asymptomatic ischemic events occur mostly due to alterations of the myocardial oxygen consumption, therefore, being preceded by elevations of the heart rate ${ }^{36}$. Because the denerved patient has no physiologic chronotropic response and has a low R-R variability, the triggering of ischemic events, documented by Holter through an underunlevelling of the ST region, after the elevation of the heart rate, becomes highly evident, giving the method a low diagnostic sensitivity, as shown in several published series and in agreement with our results ${ }^{24,37,38}$.

Myocardial thallium-201 scintigraphy under physical (ergometric test) or pharmacological (dipyridamole) stress is an important diagnostic method, often employed in the medical practice for the diagnosis of conventional coronary disease. Several reports in literature have shown a high sensitivity of this method, attaining even values of more than $90 \%$, especially in patients with proximal coronary lesions. The choice of physical or pharmacological stress has to take into consideration the individual's fitness for exercising, preferably to a maximum, to trigger myocardial ischemia that can be detected by imaging. In this analysis, we chose to use exercise scintigraphy for the purpose of 
studying the chronotropic response of these patients to exercise. Pharmacological stress was indicated for those patients who had exercise limitations or had a permanent pacemaker. In our study, the sensitivity of this test was $40 \%$ and its specificity was $96 \%$, values that are lower than those found by other groups who used pharmacological dipyridamole stress and found a 60 to $70 \%$ diagnostic sensitivity, according to the series of patients ${ }^{19,20,39.40}$. The fact that the patients had a major decrease in the chronotropic response due to the posttransplant denervation, which can be shown by the inability to reach maximum heart rate, could explain in part the low sensitivity of the method. Another important aspect to consider is the fact that the pattern of the vascular disease of the graft is frequently diffuse and affects the distal part of the vessels, leading to the so-called balanced ischemia, which may be difficult to detect by imaging diagnostic methods that compare the differences in radioisotope uptake by the different regions of the heart. The diagnostic method becomes more sensitive as the maximum stress for the triggering of ischemia is attained, and it is possible that, in this specific population, the use of myocardial dipyridamole scintigraphy could show better diagnostic sensitivity values.

The use of dobutamine stress echocardiography for the diagnosis of coronary disease has increased over the last few years and is based on the concept that high and progressive doses of dobutamine have an influence on the increase in heart labor, due to the positive inotropic and chronotropic effects. So the ischemic events are triggered as the heart rate increases. The sensitivity of this method for conventional coronary disease varied from $80 \%$ to $95 \%$, according to the series, being more sensitive for the detection of ischemias related to the anterior descending and the right coronary arteries and less sensitive in the region of the circumflex artery ${ }^{41,42}$. As for the detection of vascular disease of the graft after cardiac transplantation, the results are still controversial. Spes et al. ${ }^{43}$ obtained a $79 \%$ sensitivity and an $83 \%$ specificity with this method in the detection of vascular disease of the graft, and Derumeaux et al. ${ }^{44}$ found an $86 \%$ sensitivity and a $95 \%$ specificity in their series. In our study, the diagnostic sensitivity was $63 \%$ and the specificity was $91 \%$, with all patients who had the test done reaching maximum heart rate, thus characterizing an effective test. These patients, however, had normal ventricle function and no segmental hypocontractility on echocardiography at rest during the inclusion period of the study and, when this subgroup of patients was analyzed in other studies, a lower sensitivity was observed as well, simi- lar to the one we found. Another aspect that may influence the analysis of the diagnostic methods based on qualitative rather than quantitative criteria is the observer's experience, which makes it desirable that the analysis be performed by two observers and, on request, by a third one, if any diagnostic disagreement occurs between the first two observers. Lately, studies are under way in coronary patients to assess the impact of the findings of dobutamine stress echocardiography on the prevention of cardiac events such as heart failure, unstable angina, acute myocardial infarction, retransplantation, and death of cardiac origin. Negative test findings were related to a good prognosis in several studies, suggesting that this diagnostic method may help identify patients with a functionally important coronary disease and at risk of suffering some event in the future ${ }^{45-47}$. In patients who underwent cardiac transplantation this concept is not yet well established. In this study, when two diagnostic methods (stress echocardiography and myocardial scintigraphy) are analyzed together, considering the test as positive whenever at least one of the tests was altered, diagnostic sensitivity increases to $71 \%$. With the possibility of differentiating patients at higher risk for coronary events, especially in selected groups of patients, the use of these noninvasive diagnostic methods may become an effective option in the attempt to replace periodic angiographic evaluation in patients who undergo cardiac transplantation, but further studies with long-term follow-up are needed to confirm this proposition. In spite of its lower cost, the association of the two methods still carries some doubts regarding the therapeutic procedures to be proposed, yet signs are present indicating that normal tests and normal ventricle function may be predictive of a good prognosis.

Limitations of the study - The small number of patients (15) with angiographic documentation of their disease is a limiting factor for the analysis of the results of the noninvasive diagnostic methods, therefore requiring further studies to obtain more accurate predictive values for the employed diagnostic methods and for the confirmation of our results.

Additional information obtained from intravascular ultrasound could be useful for the detection of endothelial dysfunction occurring early in the postoperative period of transplant and might counteract the underestimation of lesions by angiography; yet, its high cost and the lack of documented knowledge of its true prognostic value have discouraged its use in the routine handling of patients. 


\section{References}

1. Lower RR, Kontos HA, Kosek JC, Sewell DH, Graham WH. Experiences in heart transplantation. Am J Cardiol 1968; 22: 766-71.

2. Thomson JG. Production of severe atheroma in a transplanted heart. Lancet 1969; 2: 1088-92.

3. Fiorelli A, Stolf N, Graziosi P, et al. Incidência de coronariopatia após o transplante cardíaco ortotópico. Rev Bras Cir Cardiovasc 1994; 9: 69-80.

4. Gao SZ, Alderman EL, Schroeder JS, et al. Clinical and laboratory correlates of accelerated coronary artery disease in the cardiac transplant patient. Circulation 1987; 76: 56-61.

5. Grattan MT, Moreno Cabral CE, Starnes VA, Oyer PE, Stinson EB, Shumway NE Eight year results of cyclosporine treated patients with cardiac transplants. J Thorac Cardiovasc Surg 1990; 99: 500-9.

6. Greenberg ML, Uretsky BF, Reddy PS, et al. Long term hemodynamic follow-up of cardiac transplant patients treated with cyclosporine and prednisone. Circulation 1985; 71: 487-94

7. Bocchi E, Vilas-Boas F, Pedrosa AA, et al. Doença coronariana após transplante cardíaco ortotópico. Arq Bras Cardiol 1994; 62: 195-200.

8. Gao SZ, Schroeder JS, Hunt AS, Billingham ME, Valentine HA, Stinson EB. Acute myocardial infarction in cardiac transplant recipients. Am J Cardiol 1989; 64: 1093-7

9. Starke RP, Mcginn AL, Wilson RF. Chest pain in cardiac transplant recipients: evidence of sensory re-innervation after cardiac transplantation. N Engl J Med 1991; 324: 1791-4

10. Halpert I, Goldberg AD, Levine AB, Kornberg R, Kelly C, Lesch M. Reinnervation of the transplanted human heart as evidenced from heart rate variability studies. Am J Cardiol 1996; 77: 180-3.

11. Gao SZ, Alderman EL, Schroeder JS, Silverman JF, Hunt SA. Accelerated coronary vascular disease in the heart transplant patient: coronary arteriographic findings. J Am Coll Cardiol 1988; 12: 334-40.

12. Valantine H, Pinto FJ, Goar FG, Alderman EL, Popp RL. Intracoronary ultrasound imaging in heart transplant recipients: the Stanford experience. J Heart Lung Transpl 1992; 11: 60-4.

13. Rickenbacher PR, Kemna MS, Pinto FJ, et al. Coronary artery intimal thickening in the transplanted heart. An in vivo intracoronary ultrasound study of immunologic and metabolic risk factors. Transplantation 1996; 61 (suppl 1): 46-53.

14. Kapadia SR, Nissen SE, Tuzcu EM. Impact of intravascular ultrasound in understanding transplant coronary artery disease. Curr Opin Cardiol 1999; 14: 140-50.

15. Rodney RA, Johnson LL. Myocardial perfusion scintigraphy to acess heart transplant vasculopathy. J Heart Lung Transpl 1992; 11: 74-8.

16. Verhoeven PPAM, Lee FA, Ramahi TM, et al. Prognostic value of noninvasive testing one year after orthotopic cardiac transplantation. J Am Coll Cardiol 1996; 28: 183-9.

17. Spes $\mathrm{CH}$, Klauss V, Mudra H, et al. Diagnostic and prognostic value of serial dobutamine stress echocardiography for noninvasive assessment of cardiac allograft vasculopathy: a comparison with coronary angiography and intravascular ultrasound. Circulation 1999; 100: 509-15.

18. Akosah KO, Mcdaniel S, Hanrahan JS, Mohanty PK. Dobutamine stress echocardiography early after heart transplantation predicts development of allograft coronary artery disease and outcome. J Am Coll Cardiol 1998; 31: 1607-14.

19. Sones FM, Shirey EK, Prondfit WL, Westcott RN. Cine-coronary arteriography. Circulation 1959; 20: 773.

20. Uretsky BF, Murali S, Reddy S, et al. Development of coronary artery disease in cardiac transplant patients receiving immunosupressive therapy with cyclosporine and prednisone. Circulation 1987; 76: 827-34.

21. Smart FW, Ballantyne CM, Cocanougher B, Farmer JA, Sekela ME, Noon GP, Young JB. Insensitivity of noninvasive tests to detect coronary artery vasculopathy after heart transplant. Am J Cardiol 1991; 67: 243-7.

22. Consenso Nacional de Ergometria. Arq Bras Cardiol 1995; 65: 191-211.

23. ACC/AHA Guidelines for exercise testing. A report of the American College of Cardiology/American Heart Association. Taske Force on Practise Guidelines. J Am Coll Cardiol 1997; 30(suppl 1): 260-315.
24. Bourdillon PDV, Broderick TM, Sawada SG, et al. Regional wall motion index for infarct and non-infarct regions after reperfusion in acute myocardial infartic comparison with global wall motion index. J Am Soc Echocardiogr 1989; 2: 398-407.

25. Agresti A. Categorical Data Analysis. New York: John Wiley \& Sons, 1990: 71-97.

26. SAS Institute Inc., SAS/STATâ User's Guide. Version 6, fourth edition, volume 1, Cary, NC: SAS Institute Inc., 1989.

27. Pennock JL, Oyer PE, Reitz BA, et al. Cardiac transplantation in perspective for the future. J Thorac Cardiovasc Surg 1982; 83: 168-77.

28. Barnhart GR, Pascoe EA, Mills AS. Accelerated coronary atherosclerosis in cardiac transplant recipients. Transplant Ver 1988; 1: 31-46.

29. Fitzgerald PJ, Goar FG, Connolly AJ, et al. Intravascular ultrasound imaging of coronary arteries. Is three layers the norm? Circulation 1992; 86: 154-8.

30. Goar FG, Pinto FJ, Aldermanl EL, Valantine HA, et al. Intracoronary ultrasound in cardiac transplant recipients. Circulation 1992; 85: 979-87.

31. Rickenbacher PR, Kemna MS, Pinto FJ, et al. Coronary artery intimal thickening in the transplanted heart. Transplantation 1996; 61: 46-53.

32. Martin TW, Gaucher J, Pupa LE, Seaworth JF. Response to upright exercise after cardiac transplantation. Clin Cardiol 1994; 17: 292-300.

33. Kao AC, Van Trigt P, Shaeffer-MCCAL GS, et al. Central and peripheral limitations to upright exercise in untrained cardiac transplant recipients. Circulation 1994; 89: 2605-15.

34. Stern S, Tzivoni D. Early detection of silent ischaemia heart disease by 24 hours eletrocardiographic monitoring of active subjects. Br Heart J 1974; 36: 481-5.

35. Epstein SE, Quyyumi AA, Bonow RO. Myocardial ischaemia: silent or symptomatic. N Engl J Med 1988; 318: 1038-43

36. Deanfield JE, Selwyn AP, Chierchia S, et al. Myocardial ischaemia during daily life in patients with stable angina: Its relation to symptoms and heart rate changes. Lancet 1983; 1: 753-8.

37. Halpert I, Goldberg AD, Levine AB, et al. Reinnervation of the transplanted human heart as evidenced from heart rate variability studies. Am J Cardiol 1996; 77 : 180-3.

38. Bigger JT, Steinman RC, Rolnitzky LM, Fleiss JL, Albrecht P, Cohen RJ. Power low behavior of RR-interval variability in healthy middle aged persons, patients with recent acute myocardial infarction, and patients with heart transplants. Circulation 1996; $93 \cdot 2142-51$.

39. Richter J, Herreros J, Serena A, Domper M, Ramirez JC, Arias R. Thallium scintigraphy in human transplants: a way to detect myocardial damage. J Heart Lung Transplant 1991; 10: 33-7

40. Smart FW, Grinstead WC, Cocanougher B, et al. Detection of transplant arteriopathy: does exercise thallium scintigraphy improve noninvasive diagnostic capabilities? Transplant Proc 1991; 23: 1189-92.

41. Segar DS, Brown SE, Sawada SG, Ryan T, Feigenbaum H. Dobutamine stress echocardiography: correlation with coronary lesion severity as determined by quantitative angiography. J Am Coll Cardiol 1992; 19: 1197-202.

42. Marcovitz PA, Armstrong WF. Accuracy of dobutamine stress echocardiography in detecting coronary artery disease. Am J Cardiol 1992; 69: 1269-73.

43. Spes CH, Mudra H, Schnaack SD, et al. Dobutamine stress echocardiography for noninvasive diagnosis of cardiac allograft vasculopathy: a comparison with angiography and intravascular ultrasound. Am J Cardiol 1996; 78: 168-74.

44. Derumeaux G, Redonnet M, Mouton S, et al. Dobutamine stress echocardiography in orthotopic heart transplant recipients. J Am Coll Cardiol; 25: 1665-72.

45. Akosah KO, Olsovsky M, Kirchberg D, Salter D, Mohanty PK. Dobutamine stress echocardiography predicts cardiac events in heart tranplant patients. Circulation 1996; 94: 283-8.

46. Lewis JF, Selman SB, Murphy JD, Mills RM, Geisen EA, Conti CR. Dobutamine echocardiography for prediction of ischemic events in heart transplant recipients. J Heart Lung Transplant 1997; 16: 390-3.

47. Spes CH, Mudra H, Schnaack SD, et al. Prognostic value of dobutamine stress echocardiography after heart transplantation. J Am Coll Cardiol 1996; 29: 290. 